\title{
Hemi-aortic arch debranching for hybrid aortic arch repair by sequential transposition of the left common carotid and subclavian arteries
}

\author{
Ludovic Canaud, MD, Frédéric Joyeux, MD, Vincent Ziza, MD, Pascal Branchereau, MD, \\ Charles Marty-Ané, MD, $\mathrm{PhD}$, and Pierre Alric, $\mathrm{MD}, \mathrm{PhD}$
}

\begin{abstract}
Objective: The aim of this study was to assess the short-term and midterm results after hemi-aortic arch debranching for hybrid aortic arch repair by sequential transposition of the left common carotid artery and of the left subclavian artery.
\end{abstract}

\begin{abstract}
Methods: From November 1998 to August 2011, 11 patients underwent a hybrid technique with supra-aortic debranching (by sequential transposition of the left common carotid artery and of the left subclavian) and simultaneous endovascular stent grafting for zone 1 lesions. There were 8 men and 3 women (mean age, $62.9 \pm 20.9$ years; range, 15-89 years). Aortic arch lesions treated included 4 complicated aortic dissections, 3 degenerative aneurysms, 2 postcoarctectomy aortic pseudoaneurysms, 1 mycotic aneurysm, and 1 traumatic transection of the arch. Four $(36 \%)$ operations were performed in an emergency setting.
\end{abstract}

Results: Endovascular exclusion success was achieved in $90.9 \%$ of the patients (type I endoleak: 1/11). One iliac artery rupture occurred intraoperatively. The 30-day mortality rate was $0 \%$. Overall actuarial survival was $82 \%$ and $71.8 \%$ at 1 and 2 years. Mean follow-up is $31 \pm 25$ months (range, 3-72 months). No instance of permanent cerebral or spinal cord ischemia was observed. Two type II endoleaks are currently observed. There was no device migration.

Conclusions: Hybrid aortic arch repair by sequential transposition of the left common carotid artery and of the left subclavian artery for zone 1 lesions provides an attractive alternative for treating hemi-aortic arch lesions in high-risk patients with minimal atherosclerotic disease in the aorta and great vessels with acceptable primary results and encouraging midterm efficacy to prevent rupture. This hybrid strategy avoiding prosthetic bypass offers several advantages over conventional repair, including the potential to treat patients who are not candidates for open repair and single-stage treatment of some pathologic conditions previously requiring 2-stage repair. (J Thorac Cardiovasc Surg 2013;145:764-7)

Repair of the aortic arch is a surgical dilemma, mainly owing to adverse neurologic sequelae ranging from $1 \%$ to $10 \%{ }^{1}$ The mortality has decreased over the past decades as various modifications of surgical technique have been introduced. Despite these recent advances, reconstruction of the aortic arch remains a challenge, particularly in elderly patients, in patients requiring emergency repair, or in those with major preexisting comorbidities. Furthermore, many patients are deemed unsuitable owing to serious comorbidities.

As a consequence, a less invasive approach would be even more favorable to patients. A combined endovascular and open approach has therefore recently been adopted as a valuable alternative, consisting in supra-aortic debranching

From the Department of Vascular and Thoracic Surgery, Arnaud de Villeneuve Hospital, Montpellier, France.

Disclosures: Authors have nothing to disclose with regard to commercial support. Received for publication Dec 6, 2011; revisions received Jan 31, 2012; accepted for publication March 12, 2012; available ahead of print April 5, 2012.

Address for reprints: Ludovic Canaud, MD, Service de Chirurgie Vasculaire et Thoracique, Hôpital A de, Villeneuve, 191 av Doyen Gaston Giraud 34090, Montpellier, France (E-mail: ludoviccanaud@hotmail.com).

$0022-5223 / \$ 36.00$

Copyright $(c) 2013$ by The American Association for Thoracic Surgery doi:10.1016/j.jtcvs.2012.03.012 and revascularization followed by stent-graft deployment. Debranching is performed to provide an appropriate landing zone for the stent graft and to preserve perfusion to the supra-aortic trunks.

In patients with zone 1 anatomy (aortic arch map proposed by Ishimaru ${ }^{2}$ ), extra-anatomic revascularization of the left common carotid artery (LCCA) and left subclavian artery (LSA) is performed with a carotid-carotid bypass, followed by a left carotid-subclavian transposition. Double transposition of the LCCA and of the LSA can also be proposed, avoiding the need of an alloplastic material bearing the risk of infection, of neck discomfort, or of dysphagia.

The aim of this study was to assess short-term and midterm results of hemi-aortic arch debranching for hybrid aortic arch repair by sequential transposition of the LCCA and of the LSA in patients deemed unsuitable for open surgery (American Society of Anesthesiologists [ASA] score $\geq 3$ ).

\author{
METHOD \\ Patients \\ Institutional review board consent was obtained. From November 1998 \\ to August 2011, 77 patients required stent-graft landing in the zones 0 to 2 \\ of the aortic arch (zone 0,14 patients; zone 1, 14 patients; zone 2, 49
}




\section{Abbreviations and Acronyms \\ ASA = American Society of Anesthesiologists \\ $\mathrm{CT}=$ computed tomograhy \\ LCCA $=$ left common carotid artery \\ LSA $=$ left subclavian artery}

patients). Among them, 14 underwent a hybrid technique with supra-aortic debranching and simultaneous endovascular stent grafting for zone 1 lesions. Eleven of them underwent sequential transposition of the LCCA and LSA. For the 3 remaining patients, extra-anatomic revascularization of the LCCA and LSA was performed with a carotid-carotid bypass, followed by a left carotid-subclavian transposition. These 3 patients were at a too high surgical risk or were deemed unsuitable for sequential transposition owing to proximal atherosclerotic lesions of the supra-aortic trunks. There were 8 men and 3 women (mean age, $62.9 \pm 20.9$ years; range, 15-89 years). All patients were unfit for open surgery because of serious comorbidities (ASA $\geq$ III). Furthermore, 4 (36\%) operations were performed in an emergency setting.

Four $(36 \%)$ patients had a complicated aortic dissection (3 dissecting aneurysms, 1 rupture of acute type B dissection). Three (28\%) patients had a degenerative aneurysm, 1 of which was ruptured. Two (18\%) patients had postcoarctectomy aortic pseudoaneurysm. One (9\%) patient had a mycotic aortic arch aneurysm. One (9\%) patient had a traumatic transection of the aortic isthmus.

All patients were considered to have an inadequate proximal landing zone for aortic arch stent-graft insertion.

\section{Surgical Approach}

All procedures took place in the operating room with the patient under general anesthesia. Debranching procedures were performed before the deployment of the stent grafts. Endovascular repair was performed in the same operating setting in all patients.

A left vertical incision was performed along the medial aspect of the sternocleidomastoid muscle and was extended vertically downward toward the manubriosternal junction. This was followed by a vertical manubriotomy. All supra-aortic branches were exposed. After systemic heparinization, the LCCA was dissected free and clamped. The vessel was divided transversely. The proximal portion was closed with a 5-0 Prolene polypropylene running suture (Ethicon, Inc, Somerville, NJ). At the next step, the brachiocephalic trunk was partially clamped and longitudinally opened, and a side-to-end anastomosis was performed. Then, the LSA was transposed into the previously transposed LCCA.

\section{Stent Graft}

Two different stent grafts were used: the Valiant device (Medtronic Vascular, Minneapolis, Minn) in 6 patients and the TAG device (W. L. Gore \& Associates, Inc, Flagstaff, Ariz) in 5 patients. The mean stent-graft diameter was $34.5 \pm 5 \mathrm{~mm}$ (range, $22-42 \mathrm{~mm}$ ).

\section{Endovascular Repair}

All patients underwent contrast-enhanced computed tomographic (CT) scan to assess the feasibility of endoluminal repair, sizing of stent grafts, and implant strategy. Suitable morphology for stent-graft placement requires a proximal aortic neck length of at least $15 \mathrm{~mm}$. Measurements from preprocedural imaging data were used to select the appropriate diameter and length of the stent graft. Devices were oversized by $10 \%$ to $20 \%$ greater than the diameter of the aortic neck to provide sufficient radial force for adequate fixation. All patients underwent preoperative CT scan of the femoral, iliac, and supra-aortic arteries to evaluate access vessels and supra-aortic trunks.
Prophylactic use of cerebrospinal fluid drainage to prevent spinal cord ischemia was not used. The endovascular devices were delivered via the common femoral artery. A 260-cm, 0.035-inch guide wire (Terumo Medical Corporation, Tokyo, Japan) was placed, via the common femoral artery, under fluoroscopic control (digital $\mathrm{C}$-arm) into the ascending aorta; a $5 \mathrm{~F}$ calibrated pigtail catheter was advanced into the ascending aorta over the guide wire. This pigtail catheter was used to exchange the guide wire for an extra-stiff guide wire (Lunderquist; Cook Medical, West Lafayette, Ind); angiography was performed through a $5 \mathrm{~F}$ pigtail catheter advanced into the ascending aorta through the LCCA. The stent graft was deployed under fluoroscopic control. A baseline angiogram was performed before and after stent-graft deployment to confirm proper position of the stent graft and complete exclusion of the lesion. Ballooning was performed selectively.

\section{Follow-up (Figure 1)}

Follow-up surveillance was performed with serial CT scans at 1 week, then at 6 and 12 months, and annually thereafter. Stent-graft migration was evaluated on a workstation analyzing CT scans with 3-dimensional reconstructions and was considered significant if measured at more than $10 \mathrm{~mm}$ between the first postoperative CT scan and another CT during the follow-up.

\section{RESULTS}

Technical success was $90.9 \%$. The mean proximal aortic diameter was $30.2 \pm 6 \mathrm{~mm}$. The mean distal aortic diameter was $31 \pm 4 \mathrm{~mm}$. The mean proximal aortic neck length was $15.9 \pm 3 \mathrm{~mm}$. The mean length of the covered aorta was $161.5 \pm 26 \mathrm{~mm}$. The mean diameter of the aneurysms was $56 \pm 14 \mathrm{~mm}$. Mean cerebral ischemic time was $12 \pm$ 6 minutes. The 30-day mortality rate was $0 \%$. Overall actuarial survival was $82 \%$ and $71.8 \%$ at 1 and 2 years. Mean follow-up is $31 \pm 25$ months (range, 3-72 months). One iliac artery rupture occurred intraoperatively and was treated by implantation of a covered stent (Wallgraft; Boston Scientific, Natick, Mass). One case of retrograde type A dissection occurred on the fourth postoperative day; the patient underwent immediate surgical conversion with ascending aortic replacement under circulatory arrest and suture between the prosthetic graft and the stent graft, which resulted in a favorable outcome. One patient with an acute mediastinitis that led to the formation of a mycotic aneurysm was treated by double transposition and endovascular aortic arch repair. She was observed for a type I endoleak because she was considered unsuitable for zone 0 debranching owing to her mediastinitis. She died of mediastinitis on the 37 th postoperative day. One patient died on the 98th postoperative day related to a cholesterol embolism syndrome leading to an ischemic colitis, transient ischemic attack, and acute renal failure. Two patients with type II endoleak, associated with a decrease in size of the aneurysm, are currently being observed. There were no instances of permanent cerebral or spinal cord ischemia or of device migration.

\section{DISCUSSION}

Repair of the aortic arch is a surgical dilemma, mainly owing to adverse neurologic sequelae ranging from $1 \%$ to 

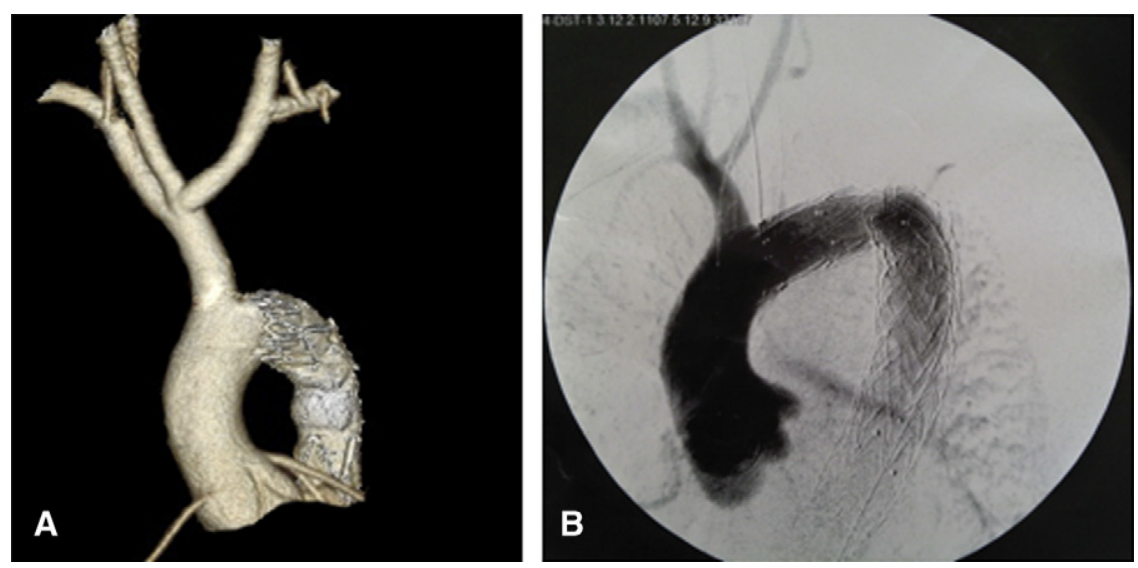

FIGURE 1. A, Postoperative computed tomographic scan after sequential transposition of the left circumflex coronary artery and of the left subclavian artery and endovascular exclusion of an acute traumatic transection of the aortic isthmus. B, Intraoperative angiogram of an endovascular exclusion of a thoracic aortic false aneurysm after transposition of the left circumflex coronary artery into the brachiocephalic trunk and of the left subclavian artery into the left circumflex coronary artery.

$10 \% .^{3}$ The surgical outcome has been improving lately. However, a recent report ${ }^{3}$ indicated that the 30 -day mortality after aortic arch surgery in the standard risk population ranges from $4 \%$ to $28.6 \%$ according to the adjunctive measures for cerebral protection used and to the mode of presentation (elective or emergency).

Our experience with hybrid arch repair ${ }^{4}$ is associated with a low rate of morbidity and mortality. Similar low rates of perioperative morbidity and mortality with hybrid arch repair have been reported by others. ${ }^{5,6}$ Potential advantages of the hybrid approach over conventional repair for arch disease are several. First, these procedures, which avoid the need for cardiopulmonary bypass and aortic crossclamping in most patients, may have advantages in high-risk patients, including the potential to offer therapy to patients who are not candidates for conventional open repair. Second, endovascular grafts can be deployed from the ascending aorta down to the level of the celiac axis, thus allowing pathologic conditions of the arch and descending aorta previously requiring either extensive single-stage repair through bilateral thoracosternotomy or 2-stage repair to be treated in a single-stage procedure. Although the endovascular option can provide distinct advantages over conventional open surgery for repair of aortic arch aneurysm, it is clear that the arch is the most challenging area for stent-graft placement. Endovascular aortic arch reconstruction, although some of these adjunctive procedures remain major operations, provides an attractive alternative for treating aortic arch diseases in high-risk patients who would otherwise be unsuitable for open repair, with acceptable primary results and encouraging midterm efficacy to prevent rupture. This technique further expands the aortic territory that can be grafted.

The main advantage of the sequential transposition of the LCCA and of the LSA is the avoidance of using prosthetic graft to maintain perfusion of the arch vessels. Long-term patency of left subclavian transposition is better than the outcome of bypasses. ${ }^{7}$ It seems intuitive that transposition of the LCCA is also associated with a better patency than carotid-carotid crossover bypass. Prosthetic graft replacement always involves the risk of infection with deleterious consequences. In addition, the potential risk of clot formation on the artificial surface of a prosthetic graft is avoided.

Careful preoperative assessment of the supra-aortic trunks is mandatory. Indeed, the presence of atherosclerotic lesions at the level of the supra-aortic trunks should preclude this surgical approach to avoid atheroembolic complications after manipulation and clamping of theses arch vessels. Atheroembolic complications remain challenging during aortic arch repair, even in case of endovascular repair. Careful manipulation of central vessels as well as keeping crossclamp times short to avoid exceeding the ischemic frame of cerebral tissue is mandatory for success. This surgical approach is of course more invasive than a carotid-carotid bypass and should be reserved for patients without atherosclerotic lesions of the supra-aortic trunks and for patients suitable for a more aggressive approach.

Kotelis and associates ${ }^{8}$ have recently performed a metaanalysis to compare the outcomes of total aortic arch transposition vs hemi-aortic arch transposition for hybrid aortic arch repair. They concluded that hemi-aortic arch transposition for hybrid aortic arch repair is associated with significantly higher primary technical failure and reintervention rates compared than total arch transposition. We disagree with their conclusion. Our experience demonstrated that hemi-aortic arch transposition can be achieved with a low mortality, a low rate of stroke, and a high technical success rate.

Retrograde type A dissection after thoracic endovascular aortic repair has been reported, ranging between $1 \%$ and 
$6.8 \%$ with a high risk of mortality. ${ }^{9}$ There are many possible mechanisms causing intimal tear during surgery, including wire and sheath manipulation in the aortic arch, lateral crossclamping of the ascending aorta, and impact forces during stent-graft deployment. However, the influence of the different stent grafts on intimal injury remains uncertain. The proximal bare spring may play a role in the development of retrograde type A dissection. Balloon remodeling to match the curved aortic arch and routine oversizing of more than $20 \%$ of the stent graft may contribute to the development of retrograde type A dissection. During surgical conversion for retrograde type A dissection, the distal anastomosis should also be performed by connecting the ascending aortic graft to the stent graft, taking deep bites in the surrounding aortic wall after removal of the proximal spring. ${ }^{10}$

Limitations of this study include patient variability within a small sample size and the retrospective study design. Another drawback of this study is the lack of comparative data to confirm that transposition is better than graft bypass.

\section{CONCLUSIONS}

Hybrid aortic arch repair by sequential transposition of the LCCA and of the left LSA for zone 1 lesions provides an attractive alternative for treating hemi-aortic arch lesions in high-risk patients with minimal atherosclerotic disease in the aorta and great vessels with acceptable primary results and encouraging midterm efficacy to prevent rupture. The main advantage of the sequential transposition of the left common carotid and subclavian arteries is the avoidance of using prosthetic graft to maintain perfusion of the arch vessels. Prosthetic graft replacement always involves the risk of infection with deleterious consequences. However, patient selection is crucial.

\section{References}

1. Griepp RB. Cerebral protection during aortic arch surgery. J Thorac Cardiovasc Surg. 2001;121:425-7.

2. Ishimaru S. Stent-grafting of the aortic arch. J Endovasc Ther. 2004;11:62-71.

3. Sundt TM 3rd, Orszulak TA, Cook DJ, Schaff HV. Improving results of open arch replacement. Ann Thorac Surg. 2008;86:787-96.

4. Canaud L, Hireche K, Berthet JP, Branchereau P, Marty-Ané C, Alric P. Endovascular repair of aortic arch lesions in high-risk patients or after previous aortic surgery: midterm results. J Thorac Cardiovasc Surg. 2010;140:52-8.

5. Gottardi R, Funovics M, Eggers N, Hirner A, Dorfmeister M, Holfeld J, et al. Supra-aortic transposition for combined vascular and endovascular repair of aortic arch pathology. Ann Thorac Surg. 2008;86:1524-9.

6. Hughes GC, Daneshmand MA, Balsara KR, Achneck HA, Sileshi B, Lee SM, et al. "Hybrid" repair of aneurysms of the transverse aortic arch: midterm results. Ann Thorac Surg. 2009;88:1882-7.

7. Cinà CS, Safar HA, Laganà A, Arena G, Clase CM. Subclavian carotid transposition and bypass grafting: consecutive cohort study and systematic review. $J$ Vasc Surg. 2002;35:422-9.

8. Kotelis D, Geisbüsch P, Attigah N, Hinz U, Hyhlik-Dürr A, Böckler D. Total vs hemi-aortic arch transposition for hybrid aortic arch repair. J Vasc Surg. 2011; 54:1182-6.

9. Dong ZH, Fu WG, Wang YQ, Guo da Q, Xu X, Ji Y, et al. Retrograde type A aortic dissection after endovascular stent-graft placement for treatment of type B dissection. Circulation. 2009;119:735-41.

10. Canaud L, Alric P, Gandet T, Albat B, Marty-Ané C, Berthet JP. Surgical conversion after thoracic endovascular aortic repair. J Thorac Cardiovasc Surg. 2011; 142:1027-31. 